\title{
Martin Carayol : La formation du canon de la nouvelle en Finlande et en Estonie
}

Thèse de doctorat

Jean Léo Léonard

\section{OpenEdition}

\section{Journals}

Édition électronique

URL : https://journals.openedition.org/efo/5397

DOI : $10.4000 /$ efo. 5397

ISSN : 2275-1947

\section{Éditeur}

INALCO

Édition imprimée

ISBN : 978-2-343-08571-5

ISSN : 0071-2051

\section{Référence électronique}

Jean Léo Léonard, «Martin Carayol : La formation du canon de la nouvelle en Finlande et en Estonie »,

Études finno-ougriennes [En ligne], 47 | 2015, mis en ligne le 05 juillet 2016, consulté le 20 septembre 2021. URL : http://journals.openedition.org/efo/5397 ; DOI : https://doi.org/10.4000/efo.5397

Ce document a été généré automatiquement le 20 septembre 2021.

\section{(c) (i) (9)}

Études finno-ougriennes est mis à disposition selon les termes de la Licence Creative Commons Attribution - Pas d'Utilisation Commerciale 4.0 International. 


\title{
Martin Carayol : La formation du canon de la nouvelle en Finlande et en Estonie
}

Thèse de doctorat

\author{
Jean Léo Léonard
}

\section{NOTE DE L'ÉDITEUR}

Directeur : Antoine Chalvin (Inalco), soutenue le 5 décembre 2013 à l'Inalco.

Doctorat de littératures et civilisations comparées.

Jury : Antoine Chalvin, Eva Toulouze, Jaan Undusk, Harri Veivo.

1 L'auteur est conscient de se trouver, en tant que chercheur en littératures et civilisations comparées, mais aussi en tant qu'enseignant dans le secondaire ou en Université, à un carrefour postmoderne où les frontières de genre littéraire, mais aussi les frontières idéologiques, politiques, socioculturelles, sont brouillées par le relativisme de la société de l'information - terme à prendre avec autant de recul critique que lorsque Guy Debord parlait de la société du spectacle, ou Jean Baudrillard de la société de consommation. Le canon en littérature ou en art a-t-il encore une raison d'être? Rend-il encore les services attendus, en tant que modèle, que norme? Notre époque se caractérise par l'explosion et l'atomisation des normes. Non pas qu'elles disparaissent : elles tendent au contraire à proliférer, sous forme de micro-avis, de nano-opinions (les tweets, les commentaires sur les blogs, etc.), mais aussi de microtâches et obligations démultipliées - le travail universitaire réduit en miettes par la mutualisation des tâches administratives en est un bel exemple. D'où la question de cerner la structure des formes canoniques en littérature, mais aussi sa réception, auprès du public. Qu'est-ce qu'un «classique du genre»? Qu'est-ce qu'un produit standard, en matière de consommation culturelle? Comment la canonicité est-elle construite par l'émetteur, mais aussi établie et reconnue par les multiples récepteurs 
(instances éducatives, critique littéraire, médias, public) ? En somme, la question relève aussi bien de la littérature comparée que de la sociologie (analyse du milieu), de la sémiotique (construction des textes, de leur forme et interprétation du sens), mais aussi des sciences politiques, car maitriser et canaliser les canons devrait permettre, serions-nous tentés de penser, d'exercer un contrôle idéologique sur les sociétés. Cependant, l'affaire est plus complexe qu'elle n'en a l'air. Le canon est davantage un phénomène émergent qu'une structure et une norme imposée verticalement. Il est polymorphe. C'est plutôt un polyèdre qu'un carré ou un tube. La lecture critique que fait Martin Carayol des manuels scolaires de littérature française est, à ce titre, édifiante: la nouvelle y est peu représentée comme forme courte narrative, et les auteurs « classiques du genre » qu'on s'attendrait à voir citer ou référencer en sont soit absents, soit mentionnés incidemment en tant que nouvellistes.

2 La notion de même de canon ne va pas tant de soi qu'on pourrait le penser : entend-on par là le modèle de référence? La tendance dominante? La forme légitime ou " acceptée » et, partant, la légitimation chère à Bourdieu? La forme ou le modèle standard ? La mode dominante d'une époque? Un moule? Un courant hégémonique? Le reflet d'une idéologie nationale ou d'une ère socioculturelle? Le meilleur moyen était de tenter de répondre à ces questions par une démarche empirique questionnant d'une part des traditions nationales littéraires, d'autre part en partant implicitement du classique schéma de la communication: pas d'émission sans réception, pas de message sans contexte et sans facteurs de bruit ou de résonance. En ce qui concerne ce premier versant empirique, Martin Carayol choisit trois traditions littéraires scandinaves depuis leur transition dans la modernité (finlandaise, estonienne mais aussi suédoise); en ce qui concerne le second versant, il s'intéresse notamment à la réception du canon en tant que modèle ou tendance - en résumé, quel est son impact réel, et dans quelle mesure les « canons reçus » sont-ils vraiment dominants?

3 Le choix de Martin Carayol de centrer sa recherche principalement sur les littératures finlandaise et estonienne est donc d'autant plus pertinent qu'on s'attendrait à ce que la nouvelle canonique, tout comme le roman canonique, soit davantage visible et analysable dans ces contrées nordiques, « jeunes nations » de l'Europe médiane, en tant que construit national, comme miroir de l'identité collective.

4 À ce titre, le chapitre sur la quête du canon de la nouvelle dans l'Estonie soviétique est riche en données et en témoignages - une mine de détails fascinants sur la sociologie de la création littéraire, sur les jeux bureaucratiques, d'envie, de carrière, et ce que l'on peut appeler le marigot de la carrière littéraire. À ce titre, la recherche de Martin Carayol déborde les études littéraires au sens strict et rejoint la sociologie qualitative par de multiples aspects de ce qui, souvent, prend la forme d'une enquête dans les archives de revues littéraires, comme une relecture attentive de Looming et des débats qui traversent cet organe de liaison des cercles littéraires reconnus ou légitimés durant l'Estonie soviétique.

5 En somme, Martin Carayol part d'une problématique qui pourrait sembler conventionnelle - pour le moins: le canon, ou norme littéraire - pour poser des questions troublantes. Qui décide du canon littéraire? Qu'est-ce que le "canon » en question? Un modèle, un parangon, un moule, une norme, une mode, un genre ou un méta-genre littéraire? Mais ne s'agit-il pas d'un roi nu parmi d'autres - car l'enquête socio-littéraire que mène Martin Carayol montre que le canon est partout et nulle part, et souvent mal représenté ou mal décrit, insuffisamment formalisé et codifié, trop 
souvent imaginé ou projeté par des individus ou par des cercles sociaux. Pis, il montre que l'impact ou l'autorité du canon n'est pas toujours effective - autrement dit, le canon, qui s'en soucie vraiment? Actuellement, d'ailleurs, le canon classique - s'il nous est permis d'user d'un tel pléonasme - s'est-il dissous dans le postmodernisme ? auquel cas la thèse devient une sorte d'essai qu'on pourrait résumer comme «à la recherche du canon perdu».

Mais il y a encore plus troublant, derrière cette enquête sur le contenu et la forme du canon dans trois littératures d'Europe septentrionale: si l'on transpose à d'autres domaines de la vie socioculturelle, et à d'autres milieux, la thèse de Martin Carayol prend un relief critique sur la légitimité et le devenir de nos normes ». L'évaluation de la recherche ne se fait-elle par en fonction de normes et de standards qui relèvent d'une forme de canonicité ? Le canon ne fait-il pas partie de l'arsenal de contraintes d'adéquation à de multiples normes, pesant sur toutes les professions intellectuelles? Servitude volontaire et canonicité seraient-elles l'une des dimensions pesant sur la création? Vivons-nous avec le canon sur la tempe - sans nous payer de mots -, ou le canon n'est-il qu'une figure idéale impotente et dérisoire, un tigre de papier ? Ou bien le canon est-il au contraire un Big Brother qui ne manque aucun de nos gestes d'écart aux conventions et aux normes circulaires dont le monde postmoderne est densément chargé ? Ou bien la créativité se nourrit-elle d'une écologie qui oscille entre la production hypercanonique, canonique, semi-canonique et cette multitude de catégories et de sous-statuts et sous-genres qu'est amené à décrire Martin Carayol, parfois de manière très détaillée (on trouvera dans sa thèse de multiples tableaux récapitulatifs des œuvres recensant les nouvelles relevant de ces sous-classes). Car cette thèse apporte également une dimension taxinomique (autrement dit, sur les critères de classement d'un observable) très appréciable.

Quoi qu'il en soit, la lecture de la thèse de Martin Carayol incite à ne plus lire les histoires de la littérature ou les manuels avec le même regard. On mesure mieux les effets de routine, de conventions, de doxa, de mode, de croyance et de désinvolture qui peuvent peser sur bien des jugements et des commentaires d'œuvres dans ces " usuels ». On savait à quel point les manuels servent presque plus à exercer l'esprit critique et à réfuter qu'à acquérir de véritables connaissances, en raison de leur conventionnalisme et de leur réductionnisme. On savait que les canons littéraires s'apparentent à des modes, voire à des idées reçues, et qu'il est dérisoire de les prendre trop au sérieux - tout au plus permettent-ils de cadrer historiquement des mouvements de pensée et des tendances générales. Mais comme souvent avec les vieux meubles, on ne regarde plus les détails d'ébénisterie, voire on se prend les pieds dedans en pestant, sans chercher à en examiner la structure - à la manière du pasteur Boggis dans la nouvelle Parson's pleasure de Roald Dahl (1959) : histoire d'un pasteur protestant, collectionneur et brocanteur averti qui, afin de faire baisser le prix d'un meuble rare et précieux, le dévalue avec tant d'insistance auprès de ses détenteurs, paysans peu au fait des subtilités de Sotheby, que ceux-ci finissent par en faire du petit bois en croyant lui faire plaisir, pendant que le brocanteur est parti garer son véhicule pour charger la pièce de musée... À en croire Martin Carayol, il serait bon de ne pas jeter le canon littéraire trop rapidement aux orties, car c'est un véritable prisme de sociologie littéraire, voire un contre-modèle qui inspire plus de créativité dans l'effort pour le contourner qu'il n'inhibe véritablement la création littéraire. Ceci dit, l'auteur montre aussi que le canon peut également être un pensum et une lutte de tous les instants pour les vrais créateurs. Mais il transcende ce genre d'approche moraliste ou 
psychologisante, pour confronter création, réception et contraintes, à la manière de Hannu Rajaniemi - mathématicien et auteur de science-fiction prodige. En somme, le canon est un catalyseur, même si parfois, c'est un catalyseur par réfraction plutôt qu'une dynamo. Le détournement du canon est sans doute ce que l'on peut faire de mieux avec lui, comme l'a brillamment montré dans l'histoire littéraire l'art d'un Isidore Ducasse et d'un Arthur Rimbaud.

8 La nouvelle, en tant que forme courte en littérature, se prêtait idéalement à cette enquête à la Columbo d'un excellent lecteur et chercheur, au style vif et intelligent, qui livre là un essai incontournable de sociologie littéraire et d'esthétique. Un essai dont on attend avec impatience la publication, voire que l'on souhaiterait voir paraître aux éditions du Seuil plutôt que chez Bordas.

\section{INDEX}

Index géographique : Estonie, Finlande

Keywords : Short Story, Literary Canon, Reception

Mots-clés : nouvelle, canon littéraire, réception

motscleset novell, kirjanduse kaanon, retseptsioon

motsclesru НОВЕЛЛа, ЛИТЕРАТУРНЫЙ КАНОН, РЕЦЕПЦИЯ

nomsmotscles Estoniens, Finnois, Français

Thèmes : littérature comparée

Index chronologique : XXe siècle 